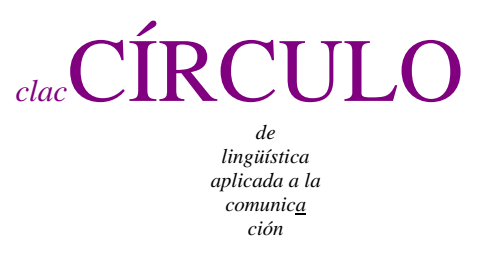

$62 / 2015$

\title{
O TÓPICO NO DISCURSO ORAL: ANOTAÇÃO E CARACTERIZAÇÃO DE DIFERENTES TÓPICOS EM CORPORA DE FALA
}

\author{
Diana Machado, Fátima Silva e Fátima Oliveira \\ Universidade do Porto \\ dianamachado03 en gmail com / mhenri en letras up pt / foliveir en netcabo pt
}

\section{Resumo}

Neste estudo, procedemos à anotação e caracterização de diferentes tipos de tópico discursivo em textos extraídos de dois corpora de fala, com incidência nos constituintes da periferia esquerda dos segmentos estudados, para i) propor uma taxonomia para a descrição semântico-discursiva destes constituintes através do recurso à ferramenta Praat; ii) caracterizar os tipos de tópicos ocorrentes; iii) discutir os resultados obtidos. Estes resultados, de natureza qualitativa e quantitativa, revelam que os falantes organizam o fluxo informacional seguindo diferentes estratégias discursivas, a articular com a estrutura sintática e sobretudo prosódica, mas também com o género do discurso oral analisado.

Palavras-chave: Tópico discursivo, corpus oral, anotação, interação sintaxe-prosódiadiscurso

Machado, Diana, Fátima Silva e Fátima Oliveira. 2015.

O tópico no discurso oral: anotaçao e caracterizaçao de diferentes tópicos em corpora de fala. Círculo de Lingüística Aplicada a la Comunicación 62, 226-253.

http://www.ucm.es/info/circulo/no62/machado.pdf

http://revistas.ucm.es/index.php/CLAC

http://dx.doi.org/10.5209/rev_CLAC.2015.v62.49505

(C) 2015 Diana Machado, Fátima Silva e Fátima Oliveira

Círculo de Lingüística Aplicada a la Comunicación (clac)

Universidad Complutense de Madrid. ISSN 1576-4737. http://www.ucm.es/info/circulo 


\section{Abstract}

The topic in oral discourse: annotation and characterization of different topics in spoken corpora

In this study, we annotate and characterize different types of discourse topic in texts extracted from two speech corpora, with emphasis on constituents of left periphery, to i) propose a taxonomy for semantic-discursive description of these constituents using Praat; ii) characterize the types of occurring topics; iii) discuss the results. These results, both qualitative and quantitative, reveal thatspeakers organize information flow following different discourse strategies, which are articulated with syntactic and prosodic structures, but also with the genre of the oral discourse at stake.

Key words: Discourse topic, oral corpus, annotation, interaction syntax-prosodydiscourse

Índice

Resumo 226

Abstract 227

1. Introdução 228

2. Metodologia 230

3. Estrutura informacional e tópico discursivo 231

3.1. Estrutura informacional 231

3.2. Tópico discursivo 234

4. Taxonomia de tópicos e anotação discursiva 236

4.1. Continuing [CONT] 239

4.2. Familiar [F] 239

4.3. Shifting [SHIFT] 239

4.3.1. Smooth-Shifting [SSHIFT] 240

4.3.2. Rough-Shiting [RSHIFT] 240 
4.4. Contrastive [C] 241

4.4.1. Contrastive 1 [C1] 241

4.4.2. Contrastive 2 [C2] 241

5.Apresentação e análise dos dados 241

5.1. Apresentação dos dados 242

5.1.1. Distribuição de tópicos nos corpora CPE-FACES e CORAL 242

5.1.2. Tipos de tópicos e sua distribuição no CPE-FACES 242

5.1.3. Tipos de tópicos e sua distribuição no CORAL 245

5.2. Análise dos resultados 247

6. Observações finais 250

Referências 251

\section{Introdução}

Este artigoapresenta os resultados de uma parte da investigação realizada no contexto do projeto COPAS ${ }^{1}$-Contraste e Paralelismo na Fala,que tem como principal objetivo a modelação da forma como a prosódia, a sintaxe e a estrutura informacional se combinam para exprimir contraste e produzir paralelismo em corpora de fala espontânea. Para avaliar essas relações de interface, uma equipa multidisciplinar determina o modo como os diferentes tipos de informação são veiculados pelos falantes no discurso oral e a ocorrência de correlações entre os domínios analisados, procedendo à anotação de corpora representativos de diferentes contextos de comunicação, com incidência na análise parcial e integrada de estruturas que envolvem a ativação, com motivação discursiva, das periferias e estruturas entoacionais a elas associadas, considerando diferentes tipos de deslocação à esquerda e à direita, estruturas clivadas e estruturas sintáticas e prosódicas com paralelismo.

\footnotetext{
${ }^{1}$ O projeto COPAS (PTDC/CLE-LIN 120017/2010) é financiado pela FCT - Fundação para a Ciência e a Tecnologia. O trabalho aqui apresentado foi realizado com o apoio de Fundos Nacionais através da FCT no âmbito do projeto COPAS.
} 
No presente estudo, concentramo-nos sobre a periferia esquerda de um conjunto de 250 segmentos discursivos, aleatoriamente extraídos de dois corpora orais de géneros distintos, 116CPE-Faces e 134 do CORAL.

O CPE-FACES - Corpus de Português Europeu Falado por Adolescentes em Contexto Escolaré um corpus de discurso espontâneo e planeado gravado em escolas secundárias portuguesas, com o objetivo inicial de "reunir um conjunto de textos falados com características situacionais diferenciadas, cuja ocorrência se assume como regular no contexto escolar português, em que os alunos participam no papel de falantes” (Mata 1995:349). Contém um total de 15h de gravações, incluindo o discurso de 2 professores e 25 alunos, e está ortograficamente transcrito.

O CORAL - Corpus de Diálogo Espontâneo ${ }^{2}$, é composto por 64 diálogos produzidos por 32 falantes divididos em 8 grupos com 4 participantes cada, a duração total de 9h e foi transcrito ortograficamente, sendo um dos objetivos centrais da sua constituição o estudo de fenómenos recorrentes no discurso oral e de difícil extração automática. Este corpusapresenta um tema comum predefinido - a orientação através de mapas -, que segue uma metodologiaMap Task ${ }^{3}$.Nestes diálogos de orientação, em que um dos participantes tem um mapa com alguns pontos de referência e uma rota traçada entre eles enquanto o outro, embora com pontos de referência, não tem nenhuma rota, devendo reconstruí-la, há tipicamente pequenas diferenças entre os dois mapas para propiciar maior interação oral.

A partir da amostra delimitada, procedemos à anotação e caracterização dos diferentes comportamentos que o tópico assume nos contextos considerados, com os seguintes objetivos: i) delimitar uma taxonomia de tópicos discursivos; ii) avaliar a adequação da taxonomia estabelecida através da anotação dos segmentos textuais analisados; iii) discutir os resultados obtidos na anotação de cada um dos corpora; iv) correlacionar esses resultados com o género discursivo desses corpora.

No sentido de dar cumprimento aos objetivos delineados, começamos por apresentar os procedimentos metodológicos adotados neste trabalho. Seguidamente, definimos o

\footnotetext{
${ }^{2}$ Informação mais pormenorizada sobre este corpusno que se refere ao seu conteúdo, participantes, recolha do dados e processo de anotação está disponível emhttps://www.l2f.inesc-id.pt/wiki/index.php/ CORAL_Corpus e http://www.clul.ul.pt/sectores/fala/coral/coral_en.php e ainda em Viana e outros (1998). ${ }^{3}$ Informação mais pormenorizada sobre a implementação desta metodologia para obtenção de corporaorais, pode ser consultada em http://groups.inf.ed.ac.uk/maptask.
} 
conceito de tópico e propomos uma taxonomia de tópicos discursivos, que serve de base à etiquetagem usada na anotação. Depois, apresentamos e discutimos os resultados obtidos na anotação, relacionando as estratégias discursivas usadas pelos falantes com as escolhas linguísticas correspondentes, para, finalmente, as correlacionarmos, ainda que de forma liminar, com os géneros dos textos orais respetivos e as áreas de interface deste projeto, a sintaxe e a prosódia.

\section{Metodologia}

A anotação dos dados selecionados foi realizada com recurso à ferramenta Praat, tendo os exemplos sido sujeitos a uma análise sequencial inicial subsequente à delimitação das fronteiras do constituinte a analisar, determinadas em interação pela prosódia, sintaxe e semântica. As fiadas apresentadas no Praat, como se pode observar na figura 1, encontram-se numeradas de 1 a 6 , com a seguinte ordenação: 1 . transcrição do exemplo; 2. tipo de constituinte; 3. tipo de construção prosódica; 4. função sintática; 5. função semântica; 6. registo de observações sobre a anotação.

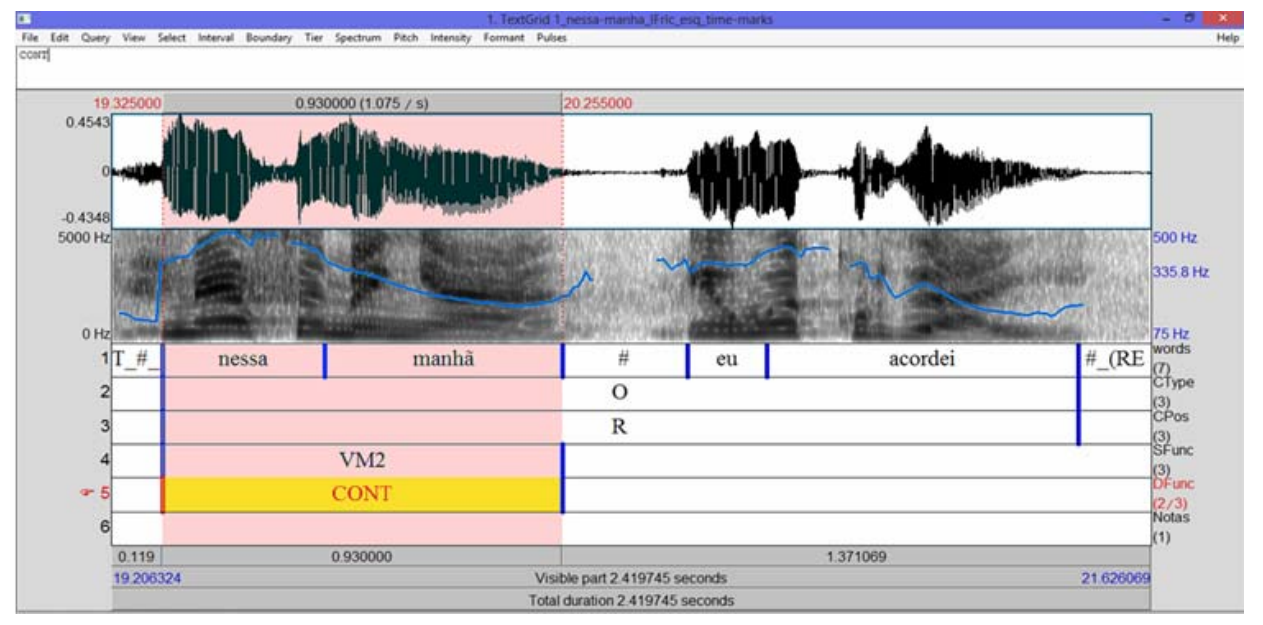

Figura 1: Visualização de um ficheiro de anotação no Praat

A anotação semântica, realizada na fiada 5, efetuou-se na sequência das anotações prosódica e sintática, tendo todas elas seguido o mesmo procedimento, que consistiu na anotação do segmento textual delimitado com recurso à audição do ficheiro áudio correspondente e ao contexto discursivo mais alargado, de modo a anotar com o maior nível de acerto possível o segmento em análise. Também com esse objetivo a anotação 
da amostra considerada neste trabalho foi antecedida da anotação, por 3 investigadores, de um subgrupo de 20 exemplos aleatoriamente selecionados em cada um dos corpora, de modo a testar e validar o conjunto de etiquetas selecionado e a metodologia de anotação proposta.

Para anotar a função semântica do constituinte que desempenha a função de tópico na periferia esquerda dos segmentos textuaisem análise, foi proposta uma taxonomia com validade teórica e exequibilidade do ponto de vista da anotação computacional. A estabilização do instrumento de anotação constituiu uma tarefa árdua, visto que se trata de uma análise pouco frequente no domínio da anotação discursiva de textos orais em português. Neste domínio, seguimos as propostas de anotação de Calhoun e outros (2005), Chafe (1987), Frascarelli e Hinterhölzl (2002), Myackykov e outros (2009), Prince (1997), Ward e Birner (2001), tendo operacionalizado a anotação nas seguintes etapas:

- análise preliminar do corpuspara observação empírica dos dados;

- revisão da literatura sobre tópico discursivo;

- proposta de uma taxonomia de tópicos discursivos;

- anotação da amostra com base na taxonomia proposta;

- correlação entre os tópicos discursivos anotados e estratégiaslinguísticas;

- análise e discussão dos resultados.

\section{Estrutura informacional e tópico discursivo}

Na secção 3, discutimos o conceito de tópico, articulando-o com o conceito de estrutura informacional, e indicamos os critérios para o estabelecimento da classificação de tópicos que sustenta a anotação realizada.

\subsection{Estrutura informacional}

O conceito de estrutura informacional (EI) tem sido entendido de formas variáveis em função do quadro teórico em que é definido e explorado. Na impossibilidade de recuperar neste âmbito todas as propostas formuladas nesse universo teórico, convocamos apenas as representações desse conceito que de forma mais nítida 
articulam a estrutura da informação com as condições nas quais se verifica o fluxo informacional, não só a nível frásico, mas sobretudo a um nível discursivo, aquele que nos concerne.

Neste domínio, o contributo do Círculo Linguístico de Praga para o tratamento da estrutura da informação é extremamente importante, salientando-se os nomes de, entre outros, Mathesius (1964), Firbas (1964) e Daneš (1974). Halliday (1967), que usou pela primeira vez o termo, considera que a estrutura informacional de um texto é constituída por uma sequência de unidades informativas que não correspondem necessariamente aos constituintes presentes na estrutura sintática, pelo que postula, ao contrário dos autores anteriores, que a informação estrutural deve ser analisada num nível distinto do da interface sintaxe-semântica (cf. Heusinger 2002, Barbosa 2005). Desta forma, o falante é livre de determinar os limites e a organização interna da estrutura informacional, visto que as unidades informacionais apresentam uma estrutura própria. Ainda segundo Halliday (1967: 200), a estrutura informacional é realizada fonologicamente pela distribuição do texto em grupos tonais. O núcleo destes grupos é composto por acentos tonais, que têm como função marcar os focos informacionais da frase. Neste sentido, a estrutura informacional é composta pela distribuição das unidades informativas (correspondentes à estrutura temática) e pela organização interna de cada uma dessas unidades, designando-se cada uma dessas estruturas, respetivamente, por estrutura temática e 'givenness'. A primeira corresponde à ordenação linear das unidades informacionais em tema-rema, enquanto a segunda refere aquilo de que se fala nessas unidades.

Lambrecht (1994), por sua vez, considera a estrutura informacional como uma componente da gramática da frase, na qual proposições como as representações concetuais dos estados de coisas

are paired with lexicogrammatical structures in accordance with the mental states of interlocutors who use and interpret these structures as units of information in given discourse contexts (Lambrecht 1994: 5).

Este autor refere-se à organização da frase como estrutura focal, ou seja, uma descrição estrutural anotada, que integra a forma fonológica e a forma semântica, e na qual os constituintes como foco e tópico são marcados. Além disso, a estrutura informacional tem em conta ainda fenómenos psicológicos, como as hipóteses que o falante coloca 
sobre os conhecimentos do ouvinte, tendo como função considerar as condições e os contextos discursivos em que as unidades de informação analisadas são produzidas (cf. Lambrecht 1994). Assim, para o autor, o estudo da estrutura informacional envolve ainda três categorias:

i. pressuposição e asserção, relacionadas com a divisão das proposições em porções que o falante assume serem ou não conhecidas pelo ouvinte;

ii. identificação e ativação dos referentes, relacionadas com as hipóteses que o falante coloca sobre o estatuto das representações mentais presentes na mente do ouvinte;

iii. tópico e foco, relacionadas com a avaliação que o falante faz da previsibilidade e não previsibilidade das relações entre as proposições e os seus elementos nas diferentes situações discursivas.

Neste enquadramento, a estrutura informacional pode manifestar-se através da prosódia, de marcadores gramaticais especiais, de constituintes sintáticos e da sua posição na frase, e de certas escolhas lexicais, constituindo uma parte da gramática da frase que combina caraterísticas semânticas, pragmáticas, prosódicas e sintáticas.

Chafe (1976) assume uma vertente de natureza mais cognitiva na sua abordagem da análise do modo como se processa o fluxo informacional, introduzindo a noção de empacotamento da informação, que é um elemento relevante para o entendimento da estrutura informacional. Neste contexto, o falante tem de atender primeiramente à forma como a mensagem é enviada e só depois à mensagem propriamente dita, situando-se a análise mais ao nível da descrição do modo como um falante acomoda o seu discurso aos estados temporários da memória do seu interlocutor e menos ao da memória de longo prazo, embora isso também se verifique sob certas condições. O empacotamento da informação está, na perspetiva de Chafe, associado a um conjunto de elementos, que têm a sua especificidade, mas contribuem para o fluxo informacional do discurso: 'givenness', contraste, definitude, sujeitos e tópicos.

Podemos, por conseguinte, concluir que é possível associar ao conceito de estrutura informacional duas distinções básicas, que permitem, na sua globalidade, uma melhor compreensão do fenómeno da estrutura da informação no discurso. Kruijff-Korbayová e 
Steedman(2003) assumem igualmente esta distinção operativa ao nível da estrutura informacional:

They all draw at least one of these distinctions: (i) A "topic/comment" or "theme/rheme" distinction between the part of the utterance that relates it to the discourse purpose, and the part that advances the discourse; (ii) a "background/contrast" or "given/new" distinction, between parts of the utterance - actually, words - which contribute to distinguishing its actual content from alternatives the context make available. (KruijffKorbayová e Steedman 2003: 251)

De facto, genericamente, os tópicos têm vindo a ser definidos como correspondendo à informação já conhecida pelos sujeitos falantes (Reinhart 1981, 1982). Na maioria dos casos, os tópicos representam informação já conhecida, mas Reinhart (1981: 73) também esclarece que sintagmas nominais com referentes idênticos podem desempenhar diferentes papéis informacionais, isto é, podem estar ou não disponíveis na mente dos falantes. Segundo esta autora (Reinhart 1982: 4) os tópicos não podem ser definidos apenas como correspondendo à informação já conhecida no discurso, dado que é possível introduzir entidades que se encontram mencionadas no discurso prévio, mas que não correspondem ao tópico do discurso em curso.

\subsection{Tópico discursivo}

Para Lambrecht, numa frase, o tópico “is the thing which the proposition expressed by the sentence is ABOUT” (1994: 118). Desta forma, um referente pode ser interpretado como tópico de uma proposição se, num determinado contexto discursivo, essa proposição expressar informação relevante que permita ao interlocutor aumentar o seu conhecimento em relação ao referente. Segundo Lambrecht (1994), o tópico encontra-se relacionado com a pressuposição pragmática, dado que

since the topic is the already established «matter of current concern» about which new information is ADDED in an utterance, for a proposition to be construable as being about a topic referent this referent must evidently be part of the pragmatic presupposition, i.e. it must already be «under discussion» or otherwise available from the context. (Lambrecht 1994: 50)

Duarte (2003), por sua vez, defende que o tópico apresenta uma função cognitiva, que tem como finalidade: 
selecionar e ativar um elemento existente na memória passiva do alocutário/leitor /ouvinte, transferindo-o para uma memória ativa em que possa ser combinado com novos elementos cognitivos introduzidos pelo comentário (Duarte 2003: 118).

Neste sentido, os referentes dos tópicos devem estar acessíveis ao locutor, podendo ser encontrados no discurso anterior ou no contexto discursivo em que o texto é produzido e interpretado (Duarte 2003: 118).

Assim, a conexões existentes entre frases permitem introduzir um referente no discurso, tornando-o, de seguida num constituinte topicalizado através de, por exemplo, relações anafóricas (Reinhart 1981: 75-76). Contudo, apesar de os enunciados se poderem apresentar ligados entre si, muitas vezes através de conectores semânticos, os seus tópicos não têm obrigatoriamente de se encontrar ligados referencialmente (Reinhart 1981: 76). Em 1982, a mesma autora propõeainda dois tipos de tópicos distintos: frásicos e discursivos. Os primeiros correspondem geralmente a uma expressão na frase, enquanto os segundos podem ser de natureza mais abstrata e correspondem a unidades maiores.

A distinção entre estes dois tipos de tópicos encontra-se também em Krifka (2001: 1) que define os tópicos discursivos como sendo "what a part of a sentence is about" e os tópicos frásicos como "what is predicated about an entity in the sentence”, sendo, em grande medida, semelhante à posição também adotada por Lambrecht (1994).

Uma forma de marcação do tópico é a deslocação à esquerda do constituinte com função de tópico. Para Reinhart (1981: 73) esta forma de construção dos enunciados pode estar relacionada com a marcação do acento prosódico ou, segundo Keenan e Schieffelin (1976) pode originar a introdução de novos tópicos. Do ponto de vista de Runge (2008: 2), esta construção frásica pode também servir para introduzir uma determinada entidade - que pode ou não já ter sido referida no discurso prévio - no discurso.

A análise de construções com deslocação à esquerda de constituintes encontra-se, também, no trabalho de Ward e Birner (2001: 29-31). Estes autores (2001: 13-18; 29-31) distinguem as construções denominadas 'preposing', em que o constituinte ocorre à esquerda e a informação está relacionada com o discurso prévio através de vários tipos de relações, como por exemplo tipo/subtipo, entidade/atributo, 
parte/todo (Ward e Birner 2001: 13), das construções com deslocação à esquerda de constituintes, nas quais a posição marcada do constituinte é ocupada por um pronome correferencial (Ward e Birner 2001: 29) e a informação veiculada pode não corresponder ao discurso prévio (Ward e Birner 2001: 30).

Prince (1997: 9), por sua vez, refere ainda que, apesar de estas construções estarem associadas à produção de tópicos contrastivos, o conceito de contraste se encontra relacionado com a ideia de saliência e com a escolha de um determinado elemento de um conjunto de alternativas. Neste sentido, a autora (1997: 9) defende que o contraste não é um resultado da posição do constituinte com função de tópico, "but rather arises when alternate when some members of some salient set are evoked and when there is felt to be opposition in what is predicated of them”.

TambémDuarte (2013: 422), no contexto da descrição das construções de topicalização, especificamente do português, estabelece uma tipologia de construções com tópicos marcados, determina as suas propriedades sintáticosemânticas e textuais específicas e analisa vários exemplos, nomeadamente extraídos de textos orais, que constituem uma base sintático-discursiva para a delimitação do contexto de ocorrência dos tópicos a seguir descritos.

\section{Taxonomia de tópicos e anotação discursiva}

À taxonomia de tópicos aplicada à anotação discursiva proposta está subjacente, pois, o princípio de que um referente pode ser interpretado como tópico de uma proposição se, num determinado contexto discursivo, ela expressa informação relevante que permite ao interlocutor aumentar o seu conhecimento em relação ao referente. Assim, o tópico assume diferentes papéis consoante as intenções e os interesses dos sujeitos falantes, pelo que diferentes tipos de tópico identificam diferentes tipos de informação veiculada pelo falante e, consequentemente, diferentes modos de organizar o discurso, sempre influenciados, entre outros fatores, pelas características da enunciação, o contexto e o próprio género textual.

A classificação proposta foi realizada em função da conceção de tópico acima exposta, bem como dos critérios relevantes para a consideração de vários subtipos de tópicos discursivos, ambos apresentados na secção 3 deste artigo, e toma como 
suporte os trabalhos de Büring (1999), Calhoun e outros (2005), Chafe (1987), Cook e Bildhauer (2011), Frascarelli e Hinterhölzl (2002), Gívon (1983), Kuno (1976), Walker e Prince (1993), assim como a análise dos dados extraídos dos corpora.

Neste contexto, a classificação proposta por Frascarelli e Hinterhölzl (2002), ela própria subsidiada pelas propostas de outros autores, entre os quais, os supracitados, serviu como ponto de partida para a delimitação de um conjunto de tipos de tópico, na medida em que este trabalho, tal como o nosso, toma como fonte de análise o discurso oral, parte do princípio de que as propriedades discursivas têm correlatos tanto no domínio fonológico como sintático, analisa constituintes da periferia esquerda com função de tópico e discrimina três tipos de tópicos, sumariados na tabela 1.

\begin{tabular}{|l|l|}
\hline \multicolumn{2}{|c|}{ Types of topics } \\
\hline Aboutness & - "what the sentence is about” (Reinhart 1981; Lambrecht 1994) \\
& $\begin{array}{c}\text { - a constituent is } \\
- \text { "newly introduced, newly changed or newly returned to" (Givón 1983) } \\
\text { - "a matter of standing and current interest or concern” (Strawson 1964) }\end{array}$ \\
\hline Contrastive & $\begin{array}{l}\text { - an element that induces alternatives which have no impact on the focus } \\
\text { value and creates oppositional pairs with respect to other topics (Kuno } \\
\text { 1976, Büring 1999) }\end{array}$ \\
\hline $\begin{array}{l}\text { Familiar } \\
\text { Continuing }\end{array}$ & $\begin{array}{l}\text { - a given or accessible constituent typically distressed and realized } \\
\text { pronominally } \\
\text { aboutness topic (Givón 1983) }\end{array}$ \\
\hline
\end{tabular}

Tabela 1: Proposta de classificação de tópicos, adaptado de Frascarelli e Hinterhölzl (2002: 1-2)

Apesar do interesse desta classificação, a nossa proposta de taxonomia de tópicos discursivos ocorrentes na periferia esquerda difere dela, na medida em que considera quatro tipos de tópicos, dois dos quais subdivididos em dois subtipos cada, como o esquema $2^{4}$ ilustra.

\footnotetext{
${ }^{4} \mathrm{~A}$ opção por manter as etiquetas correspondentes aos tópicos considerados em inglês deveu-se essencialmente ao facto de a tradução dos termos usados poder gerar alguma ambiguidade em termos do conceito exato que lhe corresponde, dado que, como vimos, há várias opções terminológicas que usam termos passíveis de serem traduzidos em português pelo mesmo termo, embora isso não signifique que
} 


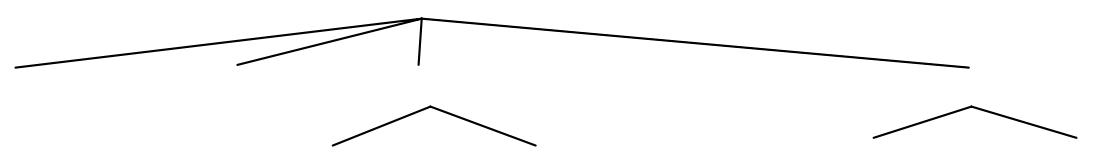

Figura 2: Taxonomia de tópicos discursivos

Esta classificação revelou-se mais adequada para a análise semântico-discursiva e a anotação dos dados no que se refere às funções desempenhadas pelos tópicos, em correlação com os contextos discursivos dos corporaconsiderados, pois permitiu operar uma caracterização mais circunstanciada dos dados, com uma especificação operativa dos diferentes tipos de tópicos ocorrentes. É nesse quadro e com o objetivo de anotar com a maior especificação possível os tópicos ocorrentes em função dos discursos em que ocorrem que propomos a separação dos tópicos familiar e continuing como tipos de

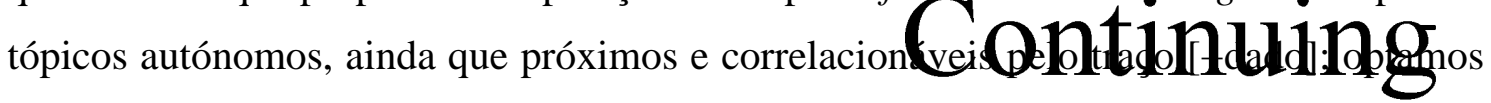
por não usar a etiqueta aboutness topic, devido ao facto de se tratar de um tipo de tópico que, pelas suas propriedades, pode incluir, de acordo com, entre outros, Givón (1983) e Reinhart (1981), tipos distintos de tópicos, nomeada e respetivamente o shifting e o continuing; e subdividimos os tópicos shifting e contrastive em dois subtipos, que permitem uma microanálise deste tipo de função, embora, para questões de uso relativo possam ser considerados em termos de macrofunção.

De seguida, apresentamos a taxonomia apresentada, definindo cada uma das etiquetas propostas, ilustradas por exemplos anotados dos corpora CPE-FACES e CORAL, correspondendo sempre a alínea a. e b. de cada exemplo ao corpus CPE-FACES e as alíneasc. e d., ao corpus CORAL. Os constituintes sublinhados marcam os segmentos aos quais foi atribuída a função de tópico.

atualizem exatamente o mesmo conteúdo. Assim, preferimos manter o termo que adotamos em vez de propormos já a sua tradução, embora seja objetivo deste trabalho vir a estabelecer, na continuidade da investigação, uma proposta terminológica que permita o uso de etiquetas em português. 


\subsection{Continuing [CONT]}

Este tipo de tópico, descrito por Givón (1983), diz respeito à continuidade de um referente no discurso, isto é, à forma que o locutor utiliza para referir uma entidade discursiva que se encontra já referida no discurso prévio, sendo manifestada frequentemente através de relações anafóricas. O exemplo (1) ilustra a ocorrência deste tipo de tópico.

(1) a. nessa manhã eu acordei

b. dessa relação nasceram três filhos

c. e depois do pico o que é que tu tens?

d. Casas simples não tenho

\subsection{Familiar $[\mathrm{F}]$}

Tal como o continuing topic, o familiar topic (Chafe 1987) encontra-se ligado à continuidade discursiva e ao estado de acessibilidade dos referentes, ocorrendo quando um determinado referente se encontra acessível no discurso prévio, como ilustra (2).

(2) a. o resto que eu vos disse sobre a atuação de DomPedro também sabemos que é verdade

b. mas antes de começarmos a trabalhar Os Lusíadas e ver de que modo é que Camõestambém ele...

c. quiosque de jornais onde é que isso está eu não tenho

d. A fonte dos domingueiros tem uma bica com uma torneira

O familiar topic distingue-se, no entanto, do continuing topic, visto queo referente daquele pode:

- não ter sido mencionado no segmento imediatamente anterior;

- requerer a realização de inferências que envolvam o conhecimento do mundo por parte do interlocutor.

\subsection{Shifting [SHIFT]}

O tipo de tópico shifting (Givón 1983) relaciona-se com a mudança discursiva, ocorrendo quando se verifica uma mudança de tópico ou a introdução de um novo tópico no discurso, que implica que o locutor abandona (momentânea ou 
definitivamente) um determinado referente e introduz outro que não se encontra (necessariamente) relacionado com o primeiro.

Segundo Walker e Prince (1993), este tipo de tópico pode ser dividido em dois subtipos: rough-shiting e smooth-shifting, distinguindo-os da seguinte forma:

A smooth-shift tends to occur when the speaker has started talking about a new entity and it is doing so in a way that indicates that s/he will continue talking about that entity, while a rough-shift tends to occur when the speaker has started talking about a new entity, butis only doing so momentarily (Walker e Prince 1993: 2-3).

A sua distinção na análise dos dados permite especificar demodo mais claro a forma como a informação é organizada ao longo do discurso nos casos em que a periferia da esquerda tem como função indicar mudança de tópico.

\subsubsection{Smooth-Shifting [SSHIFT]}

O tópicosmooth-shifting ocorre quando o falante introduz uma nova entidade no discurso de modo a indicar que possui intenções de continuar a referi-la, como se ilustra em (3).

(3) a. Dom Sancho quarto entre filhos que teve teve alguns ilegítimos

b. do Lemos é uma pessoa que é mestre em artes poéticas contos instrumentos de corda...

c. e os rochedos escarpados tens?

d. Em relação ao globo enorme onde está

\subsubsection{Rough-Shiting [RSHIFT]}

No caso do tópico rough-shifting, a sua ocorrência verifica-se quando o falante refere de forma momentânea uma nova entidade no discurso, como é ilustrado por (4).

(4) a. em Castela a certa altura houve um problema

b. depois do maridonão temos nada

c. quando chegares ao alto do alandroal vais-te dirigir para o esconderijo de piratas ferozes

d. Serro da vala mágica tens isso 


\subsection{Contrastive [C]}

Este tipo de tópico encontra-se relacionado com a expressão de contraste no discurso, ocorrendo quando se verifica a presença de um valor contrastivo ou a existência de um elemento que induz um conjunto de alternativas.

Da mesma forma que o tópico shifting, o tópico constrastivepode ser dividido em dois subtipos, tendo em conta os elementos e a forma como o contraste é realizado. Esta subdivisão permite uma maior especificação em termos da classificação das funções que o tópico desempenha, estando associada ao modo como se contrasta a informação.

\subsubsection{Contrastive 1 [C1]}

O tópico C1 (Kuno 1976; Büring 1999) representa um contraste explícito entre dois elementos (por exemplo x ou y), como se pode observar em (5).

(5) a. para mim é um herói mas para você não deve ser

b. o que fizeram a mim também já fizemos a eles

c. pagodes chineses não mas acho que irrigado é sinónimo de fértil portanto podes considerar a mesma coisa

d. Bosque queimado aqui não tenho mas tenho porto delgado tens

\subsubsection{Contrastive 2 [C2]}

Por sua vez, o tópico C2 (Calhoun e outros 2005) salienta um determinado elemento de um conjunto de alternativas presente no contexto ou no conhecimento do interlocutor, como se exemplifica em (6).

(6) a. e entre as damas que a acompanhavam todas elas de boas famílias vinha D. Inês de Castro

b. Dom Sancho quarto entre filhos que teve teve alguns ilegítimos

c. pronto depois para cima deve haver uns caminhos esburacados

d. Portanto se vais andando para a direita passas por cima das casas juntas

\section{Apresentação e análise dos dados}

Na sequência da apresentação da metodologia e etiquetagem usadas na anotação dos dados do CPE-FACES e CORAL, passamos, seguidamente, a uma apresentação dos resultados obtidos, seguida da sua análise. 


\subsection{Apresentação dos dados}

A análise dos dados foi realizada tomando em consideração as seguintes variáveis: i) tipos de tópicos e sua distribuição nos corpora CPE-FACES e CORAL; ii) tipos de tópicos e sua distribuição no CPE-FACES, iii) tipos de tópicos e sua distribuição no CORAL.

\subsubsection{Distribuição de tópicos nos corpora CPE-FACES e CORAL}

Em termos globais, a distribuição das 250 ocorrências de tópicos nos dois corpora correspondeu, quantitativa e qualitativamente, aos seguintes resultados: 69 tópicos continuing; 63 tópicos familiar; 35 tópicos shifting, dos quais 20 são soft-shifting e 15, rough-shifting; 83 tópicos contrastive, subdivididos em 61 ocorrências de C1 e 22 de C2. Assim, relativamente à análise global dos dois corporaverifica-se que $28 \%$ dos exemplos analisados foram marcados como continuing, 25\% comofamiliar, 14\% como shifting e 33\%, comocontrastive, o que permite fazer a seguinte ordenação deocorrências em termos ascendentes: $C-C O N T-F-S H I F T$. O gráfico 1 ilustra esta distribuição.

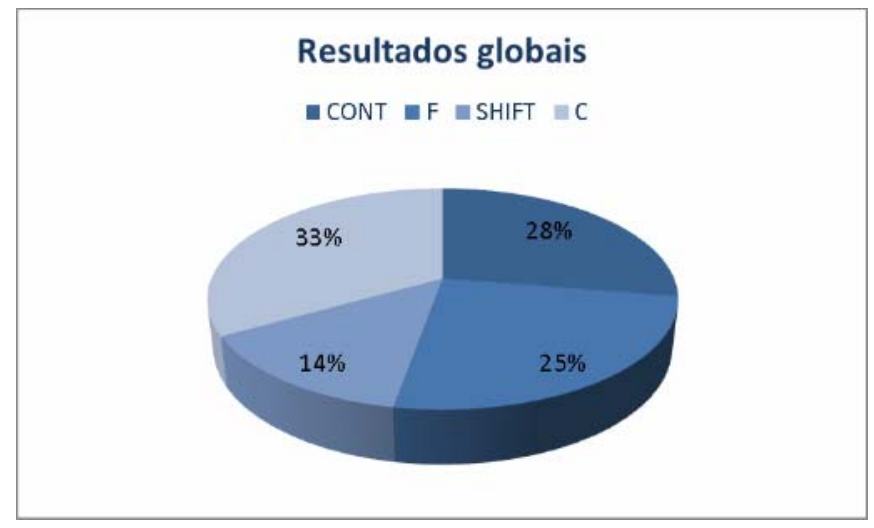

Gráfico 1: Distribuição percentual global de tópicos

\subsubsection{Tipos de tópicos e sua distribuição no CPE-FACES}

No CPE-FACES, os 116 segmentos analisados correspondem à seguinte distribuição dos tópicos: 19 tópicos continuing; 28 tópicos familiar; 25 tópicos shifting, dos quais 14 são soft-shifting 1, 11, rough-shifting; 44 tópicos contrastive, subdivididos em 33 ocorrências de C1 e 11 de C2. Assim, em termos percentuais, 38\% das ocorrências 
foram marcadas como contrastive, 22\% como shifting, 24\% como familiar e os restantes $16 \%$ foram marcados como continuing. No que diz respeito aos subtipos de shifting, verificou-se que 58\% das ocorrências foram marcadas como smooth-shifting, enquanto os outros $42 \%$ foram marcados como rough-shifting. Relativamente aos subtipos definidos para o tipo de tópico contrastive observou-se que $75 \%$ das ocorrências correspondem ao contrastive 1 e as restantes $25 \%$ correspondem ao contrastive 2 . Os gráficos 2-4 ilustram estes resultados, que organizam as ocorrências de tópicos na sequência da direita para a esquerda: C [C1 - C2]- F - SHIFT [SSHIFT - RSHIFT] CONT. Neste contexto, note-se a recorrência mais notória dos tópicos que marcam contraste, com uma clara dominância das ocorrências de C1 em detrimento de C2, tendência que não se verifica na ocorrência dos subtipos de shifting, cuja distribuição é percentualmente mais próxima.

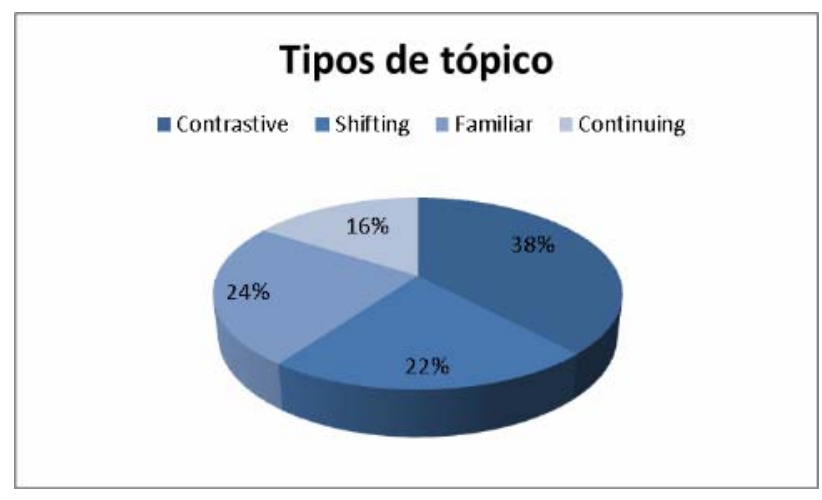

Gráfico 2: Tipos de tópicos no CPE-FACES

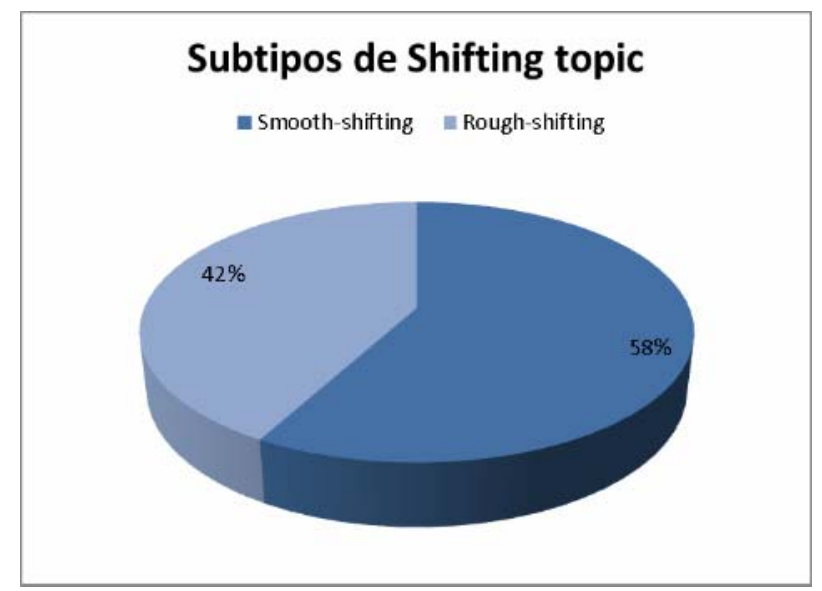

Gráfico 3: Subtipos detópicos shiftingno CPE-FACES 


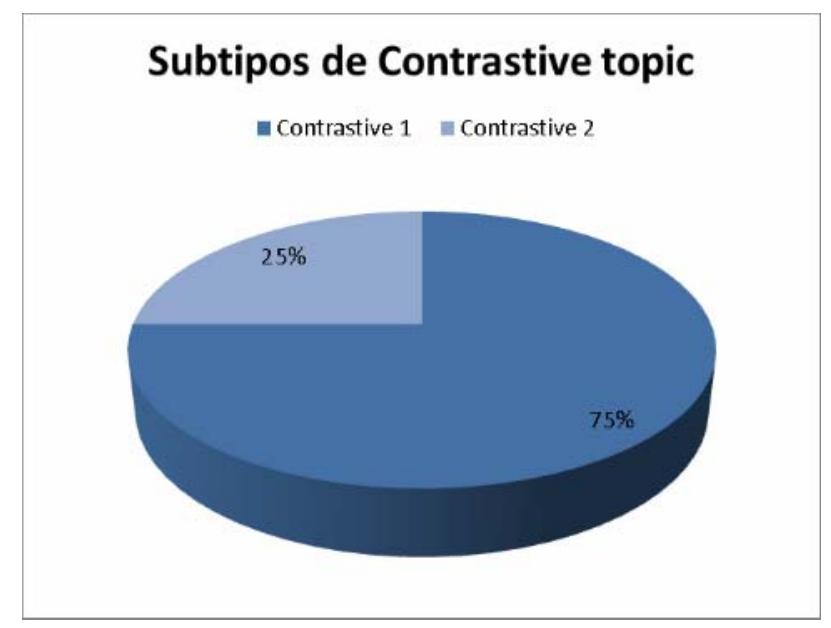

Gráfico 4: Subtipos de tópicos contrastive no CPE-FACES

As estruturas linguísticas associadas à delimitação dos tópicos neste corpus são dominantemente as que a seguir se apresentam

\begin{tabular}{|c|c|c|}
\hline \multicolumn{3}{|c|}{ Tópicos discursivos e marcadores linguísticos } \\
\hline Tópico & Marcadores linguísticos & Exemplos \\
\hline CONT & $\begin{array}{l}\text { Sintagmas preposicionais } \\
\text { Relações anafóricas }\end{array}$ & $\begin{array}{l}\text { Nessa manhã }[\ldots] / \text { Nesse dia }[\ldots] \\
\text { Isso tudo }[\ldots] / \text { Isto }[\ldots]\end{array}$ \\
\hline $\mathbf{F}$ & Sintagmas nominais & O resto $[\ldots] /$ Camões também ele $[\ldots]$ \\
\hline SSHIFT & Contexto & $\begin{array}{l}\text { posteriormente teriam de ser lembradas vezes } \\
\text { sem conta, bom, sobre Inês [...]/ [...] vamos } \\
\text { ao trabalho, eu só quero que vocês se } \\
\text { lembrem de uma expressãozinha }\end{array}$ \\
\hline RSHIFT & Contexto & $\begin{array}{l}\text { narrativas em que este amor é lembrado, } \\
\text { quanto ao beija-mão [...]/ [...] essa precisão, } \\
\text { trinta segundos é fantástica, quem é que falta, } \\
\text { ninguém e as faltas de atraso marco-as todas }\end{array}$ \\
\hline C 1 & $\begin{array}{l}\text { Conjunções } \\
\text { Sintagmas preposicionais }\end{array}$ & $\begin{array}{l}\text { se se amavam ou não }[\ldots] \text { / }[\ldots] \text { têm } \\
\text { expressão ou não } \\
\text { Ao colo }[\ldots] \text { e na mão }[\ldots] / \text { um na rua }[\ldots] \\
\text { outro na cama }\end{array}$ \\
\hline C 2 & Sintagmas preposicionais & $\begin{array}{l}\text { e entre as damas que a acompanhavam } \\
{[\ldots] /[\ldots] \text { para ti }}\end{array}$ \\
\hline
\end{tabular}

Tabela 2:Tópicos discursivos e marcadores linguísticos no CPE-FACES 


\subsubsection{Tipos de tópicos e sua distribuição no CORAL}

No CORAL, os 134 segmentos analisados correspondem à seguinte distribuição dos tópicos: 50 tópicos continuing; 35 tópicos familiar; 10 tópicos shifting, dos quais 6 são soft-shifting e 4, rough-shifting; 39 tópicos contrastive, subdivididos em 28 ocorrências de C1 e 11 de C2. Por conseguinte, neste corpus, 37\% das ocorrências foram marcadas como continuing, 29\% como contrastive, 26\% como familiar e 7\% como shifting. Relativamente aos subtipos considerados para o shifting, foi possível observar que $60 \%$ das ocorrências foram marcadas como smooth-shifting e as restantes $40 \%$ foram marcadas como rough-shifting. Quanto aos subtipos definidos para o contrastive, foram marcadas $72 \%$ das ocorrências como contrastive 1 e $28 \%$ como contrastive 2. Os gráficos 5 - 7esquematizam estes resultados, cuja ordenação em termos de recorrência é a seguinte: CONT - C [C1 - C2] - F - SHIFT [SSHIFT - RSHIFT]. Neste corpus, verifica-se uma dominância da ocorrência de tópico continuing, manifestando-se, relativamente à distribuição dos subtipos de shifting e contrastive a mesma tendência referida para os resultados obtidos no corpus CPE-FACES, isto é, ocorrência maioritária de C1 em detrimento de C2, nos casos de marcação de contraste, e uma distribuição mais aproximada ao nível dos subtipos de SHIFT.

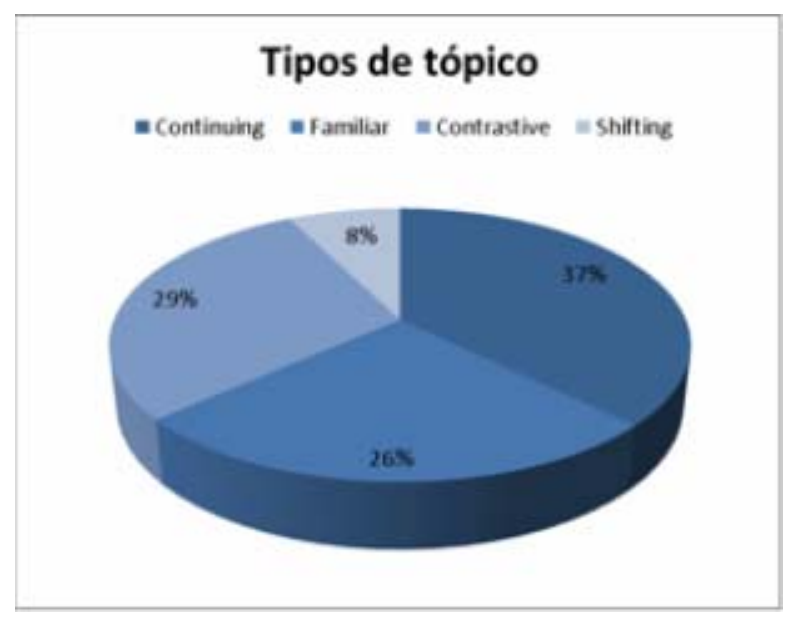

Gráfico 5: Ocorrência de tópicos no CORAL 


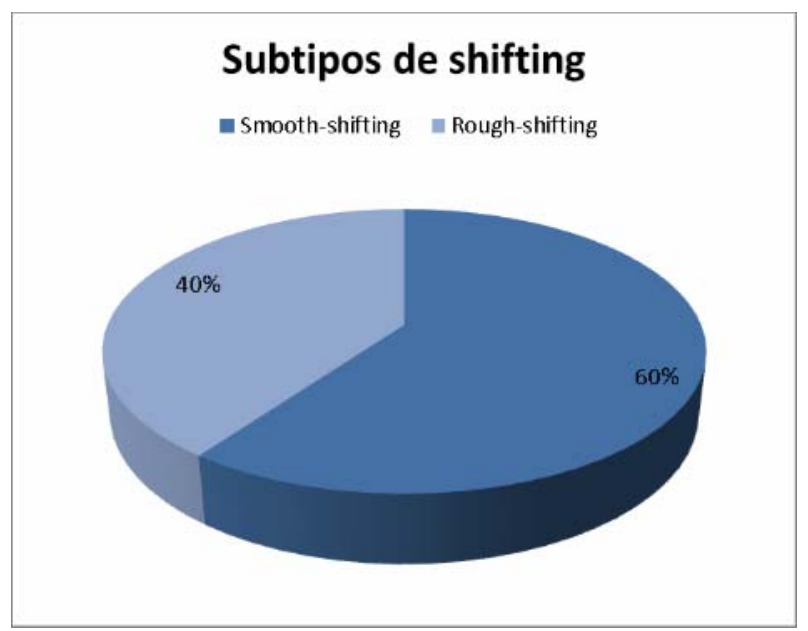

Gráfico 6: Subtipos de tópicos shifting no CORAL

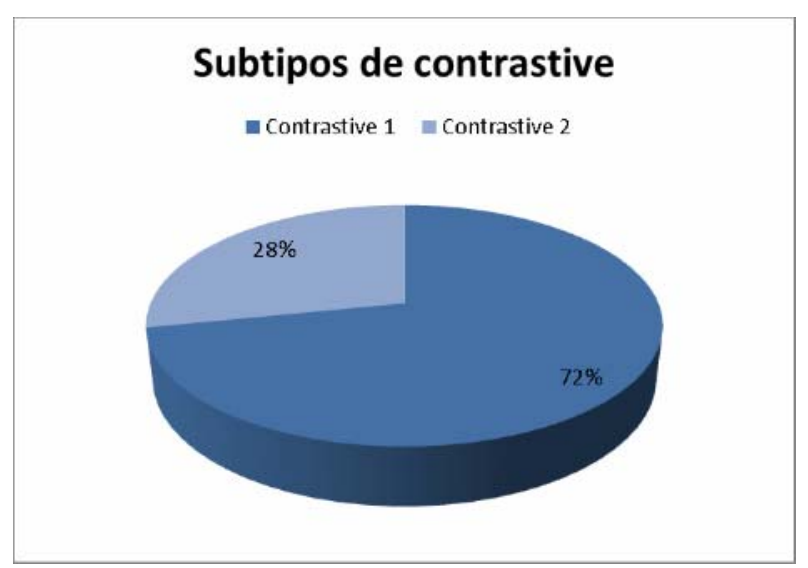

Gráfico 7: Subtipos de tópicos contrastive no CORAL

As estruturas linguísticas associadas à delimitação dos tópicos neste corpus são dominantemente as que se apresentam na tabela 3.

\begin{tabular}{|c|l|l|}
\hline \multicolumn{3}{|c|}{ Tópicos discursivos e marcadores linguísticos } \\
\hline Tópico & \multicolumn{1}{|c|}{ Marcadores linguísticos } & \multicolumn{1}{c|}{ Exemplos } \\
\hline CONT & $\begin{array}{l}\text { Sintagmas preposicionais } \\
\text { Sintagmas nominais }\end{array}$ & $\begin{array}{l}\text { nesse canto tenho um outro uma outra coisa } \\
\text { que é o esconderijo secreto } \\
\text { poste telefónico não se encontra aqui portanto }\end{array}$ \\
\hline F & Sintagmas nominais & $\begin{array}{l}\text { fos domingueiros tem uma bica com } \\
\text { uma torneira }\end{array}$ \\
\hline SSHIFT & Contexto & $\begin{array}{l}\text { Ah não espera que isto é o fim espera aí o } \\
\text { início eucaliptos jovens estás a ver o início a } \\
\text { cruzinha do início [...] }\end{array}$ \\
\hline
\end{tabular}




\begin{tabular}{|c|l|l|}
\hline RSHIFT & Contexto & $\begin{array}{l}\text { passagem de nível herdade da achada } \\
\text { mármores de esmoriz controle anti-aéreo } \\
\text { automóvel avariado lage xistosa sede do } \\
\text { movimento anti-fascista globo grande postes } \\
\text { de telecomunicações outeiros escalvados } \\
\text { cascata das grutas [...] [...] agora posso } \\
\text { respirar? }\end{array}$ \\
\hline $\mathbf{C ~} \mathbf{1}$ & $\begin{array}{l}\text { Conjunções } \\
\text { Sintagmas preposicionais }\end{array}$ & $\begin{array}{l}\text { pagodes chineses não mas acho que irrigado é } \\
\text { sinónimo de fértil portanto podes considerar a } \\
\text { mesma coisa } \\
\text { entre a ponte não concluída e as águas santas } \\
\text { existe então o barco pirata não é }\end{array}$ \\
\hline $\mathbf{C ~ 2}$ & Sintagmas preposicionais & $\begin{array}{l}\text { por baixo um bocadinho à direita quer dizer } \\
\text { há uns rochedos escarpados e há a atalaia da } \\
\text { serra e ainda mais para baixo riacho sujo e } \\
\text { depois no fundo da folha }\end{array}$ \\
\hline
\end{tabular}

Tabela 3:Tópicos discursivos e marcadores linguísticos no CORAL

\subsection{Análise dos resultados}

Da apresentação dos dados analisados decorre a verificação de algumas diferenças entre os dois corpora analisados no que se refere à distribuição dos tópicos e em estruturas da periferia esquerda e à sua frequência, mas também em relação às estruturas linguísticas que os materializam.

Recuperando a hierarquia estabelecida, observamos que existe uma variação na distribuição dos tópicos em função do corpus:

$$
\begin{aligned}
& \square \text { CPE-FACES: C [C1 - C2] - F - SHIFT [SSHIFT - RSHIFT] - CONT } \\
& \square \text { CORAL: CONT - C [C1 - C2] - F - SHIFT [SSHIFT - RSHIFT] }
\end{aligned}
$$

Essa distinção é correlativamente observável em termos da quantificação das ocorrências de cada um dos tipos de tópico nos dois corpora, como o gráfico 8 permite sintetizar. 


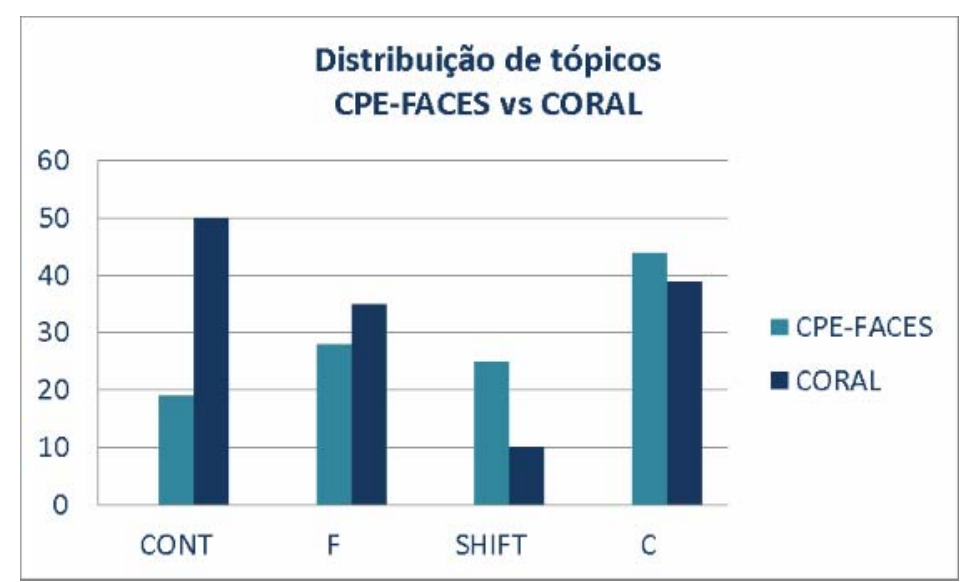

Gráfico 8: Comparação da distribuição dos tópicos no CPE-FACES e CORAL

No que diz respeito à comparação das estruturas linguísticas associadas a cada um dos tipos de tópico considerados, concluímos que há uma tendência para um uso frequente do mesmo tipo de estruturas nos dois corpora, excetuando uma variação ocorrente ao nível dos tópicos continuing e familiar.Há tipicamente uma relação entre a expressão do tópico e a estratégia escolhida pelo locutor para a sua expressão linguística, pelo que é possível encontrar determinadas regularidades ou categorias associadas a esses tópicos, verificando-se que, nos casos em que há mudança de tópico, o contexto discursivo surge como um fator determinante para a sua marcação.

Por outro lado, observando os dados de forma global, verifica-se uma determinada tendência para a hierarquia dos tópicos na organização da informação discursiva, que corresponde igualmente, em grande medida, à organização da informação discursiva em função dos parâmetros discursivos da interação.

Assim, esta distinção ao nível da distribuição dos tópicos deve ser correlacionada em grande medida com as próprias características enunciativas dos discursos produzidos bem como o contexto situacional em que ocorrem. Neste quadro, o tópico apresenta uma função cognitiva que permite transferir um determinado referente da memória passiva para a memória ativa do interlocutor, de forma a ser possível combiná-lo com outros elementos que são introduzidos pelo comentário. Os referentes dos tópicos devem, assim, estar acessíveis ao locutor, podendo ser encontrados no discurso anterior ou no contexto discursivo em que o texto é produzido e interpretado (cf. Duarte 
2003: 118). A forma como essa relação se estabelece determina o modo como o fluxo informacional se organiza e fica acessível aos interlocutores.

Desta forma, o CPE-FACES é produzido num contexto de sala de aula, marcado por um discurso de certa forma espontâneo, pela frequente interação entre os falantes e pelo relato de experiências e a exposição de conteúdos. Estes fatores originam a existência de diversas marcas linguísticas como a frequente mudança de tópico, que motiva quer a introdução de novos tópicos a meio da conversação (e a sua possível retoma posterior), quer a presença de vários contrastes ao longo do discurso. Os efeitos que advêm do contexto de produção deste corpus são visíveis através da marcação prosódica do constituinte com função de tópico, de algumas mudanças contextuais, de retomas anafóricas e ainda de alguns conetores (nomeadamente os que induzem contraste) ${ }^{5}$.

O CORAL, por sua vez, apresenta-se como uma tarefa de orientação, caracterizado por um discurso espontâneo e pela frequente interação entre os interlocutores, bem como pela existência de um guião de orientação espacial que condiciona toda a conversação. Estas caraterísticas motivam a manutenção frequente do tópico, a introdução de novos tópicos no meio do discurso (sem retoma posterior) e o contraste centrado em elementos deíticos. Os efeitos provocados pelas condicionantes da interação são percetíveis através da marcação prosódica do tópico, de alguns conetores e da frequente ocorrência de deíticos espaciais.

A anotação semântica dos dados fornece ainda informações relevantes sobre a forma como a informação se encontra organizada ao longo do discurso, nomeadamente em termos das funções desempenhadas por estruturas de tópico na periferia esquerda, que evidenciam a correlação entre estratégia linguísticaeestratégia comunicativa, constituindo por si um procedimento autónomo de anotação. No entanto, a sua execução, tratando-se de discurso oral, implica sempre que se atenda a questões de prosódia, como se explicitou nos procedimentos de anotação explicitados na secção 2, bem como à estrutura sintática. Trata-se de estabelecer uma correlação entre os dados das três áreas envolvidas sem, no entanto, as sobrepor em termos de análise específica.

\footnotetext{
${ }^{5}$ Embora este trabalho não tenha como objetivo a análise da articulação entre dados da prosódia, da sintaxe e da semântica, a convicção de que eles estão relacionados decorre de um trabalho já desenvolvido pela equipa multidisciplinar do COPAS, no qual se estabelecem os parâmetros de anotação prosódia, sintática e semântica de estruturas de tópico em análise neste trabalho (cf. Mata e outros 2013).
} 


\section{Observações finais}

Neste estudo, ocupámo-nos da caracterização e distribuição de um conjunto de tópicos ocorrentes em estruturas da periferia esquerda, a partir dos dados extraídos de dois corpora, CPE_FACES e CORAL. Através da anotação dos dados, à qual esteve subjacente a formulação de uma taxonomia de tópicos, bem como preocupações de natureza metodológica e teórica, procuramos atender, entre outras, às seguintes questões: validação da metodologia e das etiquetas usadas na anotação;distribuição dos tópicos em cada um dos corpora;correlação dos resultados obtidos nos dois corpora; contributo da análise dos formatos e funções dos tópicos da periferia esquerda para a compreensão do fenómeno da organização informacional do texto.

Assim, a classificação proposta para análise dos dados mostrou-se produtiva na discriminação dos tópicos e respetiva distribuição, tendo sido considerados quatro tipos de tópicos - CONT, F, SHIFT, C -, com a subdivisão destes últimos em dois, respetivamente SSHIFT/RSHIFT e C1/C2. Essa análise mostrou que as estratégias de organização do discurso são variadas, o que ficou patente na hierarquia de uso dos tópicos em análise, e que os dois corpora apresentam uma hierarquia de ocorrências distinta, devedora, em grande medida, dos géneros dos discursos orais considerados, que, pela sua distinção ao nível da funcionalidade e da composicionalidade, implicam divergências no estilo.

Esta hipótese deverá ser testada com recurso a mais dados, pelo que seria vantajoso alargar este modelo a outros corpora para validar a tendência evidenciada. Por outro lado, essa tendência deve ser apoiada por uma análise de interface com a sintaxe e a prosódia, como é objetivo do projeto, no sentido de corroborar e possibilitar uma análise mais aprofundada do conteúdo e modo de organização do texto. 


\section{Referências}

Barbosa, J. (2005): Foco e Tópico: algumas questões terminológicas. Em: Rio-Torto, G. e outros (eds.),Estudos em homenagem ao Professor Doutor Mário Vilela. Vol. I. Porto: Faculdade de Letras da Universidade do Porto (FLUP), 339-351.

Büring, D. (1999): Topic. Em: Bosch, P. y R. Van der Sand (eds.),Focus - Linguistic Cognitive and Computational Perspectives. Cambridge: CUP, 142-165.

Calhoun, S.e outros (2005): A Framework for Annotating Information Structure in Discourse. $<$ http://groups.inf.ed.ac.uk/switchboard/infostruc.pdf > . Acedido em 02-08-2013.

Chafe, W. (1976): Givenness, contrastiveness, definiteness, subjects, topics, and point of view. Em: Charles, N. L. (ed.), Subject and Topic. Nova Iorque: Academic Press, 25-55.

Chafe, W. (1987): Cognitive constraints on information flow. Em: Tomlin, R. S. (ed.), Coherence and grounding in discourse. Amsterdam: Benjamins, 21-51.

Cook, P. e F. Bildhauer (2011): Annotating Information Structure. The case of topic. Em: Dipper, S. e H. Zinsmeister (eds.), Beyond Semantics. Corpus-based Investigations of Pragmatic and Discourse Phenomena. Bochum: Universidade de Bochum, 45-56.

Daneš, F.(1974): Functional sentence perspective and the organization of the text. Em:Daneš, F. (ed.),Papers on Functional Sentence Perspective. Prague: Academia/The Hague: Mouton, 106-128.

Duarte, I. (2003): Aspectos linguísticos da organização textual. Em: Mateus, $M^{a}$ H. e outros, Gramática da Língua Portuguesa. Lisboa: Caminho, 87-123.

Duarte, I. (2013):Construções de Topicalização. Em: Raposo, E. B. P.,Gramática do Português. Vol. I. Lisboa: Fundação Calouste Gulbenkian, 401-426.

Firbas, J. (1964): On Defining the Theme in Functional Sentence Analysis.Travaux de Linguistique de Prague 1, 267-280.

Frascarelli, M. e R. Hinterhölzl (2002): Types of topics in German and Italian. Em: Winkler, S. e K. Schwabe (eds.), On Information Structure, Meaning and Form. Amsterdam/Philadelphia: Benjamins, 1-29.

Givón, T. (1983): Topic Continuity in Spoken English. Em: Givón, T. (ed.),Topic Continuity in Discourse: A Quantitative Cross-Language Study. Amsterdam/ Philadelphia: Benjamins, 347-363. 
Halliday, M. A. K. (1967): Notes on transitivity and theme in English. Part 2.Journal of Linguistics 3, 199-244.

Heusinger, K.von(2002): Information structure and the partition of sentence meaning. Em: Hajicová, E.e outros (eds.), Travaux du Cercle Linguistique de Prague. Prague Linguistic Circle Papers 4. Amsterdam/Philadelphia: Benjamins, 275-305.

Keenan, E. O. e B. Schieffelin (1976): Topic as a discourse notion: a study of topic in the conversations of children and adults. Em:Li, C. (ed.),Subject and Topic, Nova Iorque: Academic Press, 335-384.

Krifka, M. (2001): Topik und Fokus. Humboldt-Universität. Wintersemester 2001/02. $<$ http://amor.cms.huberlin.de/ h2816i3x/Lehre/2001_HS_TopikFokus/TopikFo.pdf > .Acedido em 28-092013.

Kruijff-Korbayová, I. e M. Steedman (2003): Discourse and information structure. Journal of Logic, Language and Information 12(3), 249-259.

Kuno, S. (1976): Subject, theme, and the speaker's empathy - A reexamination of relativization phenomena. Em: Li, C. (ed.), Subject and Topic. Nova Iorque: Academic Press, 1-29.

Lambrecht, K. (1994): Information Structure and Sentence Form - Topic, Focus and the Mental Representations of Discourse Referents. Cambridge: CUP.

Mata, A. I. (1995): Apresentação Preliminar do CPE FACES: um 'Corpus de Português Europeu Falado por Adolescentes em Contexto Escolar’ para o Estudo da Prosódia dos Estilos de Fala. Em: Nascimento, M ${ }^{a}$ F. B e outros (eds.),Actas do XI Encontro Nacional da Associação Portuguesa de Linguística. Vol. I. Lisboa: Colibri, 349-358.

Mathesius, V. (1964): On some problems of the systematic analysis of grammar. Travaux $d u$ Cercle Linguistique de Prague 6, 95-107.

Myackykov, A. e outros (2009): Attention and syntax in sentence production: A critical review. Em: Discours [En ligne] 4. < http://discours.revues.org/7594 >.Acedido em09-07-2013.

Prince, E. F. (1997): On the functions of left-dislocation in English discourse. Em: Kamio, A. (ed.), Directions in Functional Linguistics. Amsterdam/Philadelphia: Benjamins, 117143. 
Reinhart, T. (1981): Pragmatics and linguistics: an analysis of sentence topics.Philosophica 27, 53-94.

Reinhart, T. (1982):Pragmatics and Linguistics: An Analysis of Sentence Topics. Bloomington (Ind.): Indiana University Linguistic Club.

Runge, S. (2008): Left-dislocations and their contribution to information structure. Paper presented at the "Biblical Greek Language and Linguistics" of the SBL Annual Meeting. < http://www.ntdiscourse.org/docs/Runge_L-dis_SBL.pdf > . Acedido em 23-08-2013.

Strawson, P. F. (1964): Intention and convention in speech acts.The Philosophical Review 73(4), 439-460.

Viana, Ma C. e outros (1999): Apresentação do Projecto CORAL-Corpus de Diálogo Etiquetado. Em: Marrafa, P. e $\mathrm{M}^{\mathrm{a}}$ A. Mota (eds.), Linguística Computacional: Investigação Fundamental e Aplicações. I workshop sobre linguística computacional da APL, FLUL, maio de 1998. Lisboa: Edições Colibri, 337-345.

Walker, M. A. e E. F. Prince (1993):A bilateral approach to givenness: a hearer-status and a centering algorithm. Em: Fretheim, T. e J. Gundel (eds.),Reference and referent accessibility. Amsterdam/Philadelphia: Benjamins, 291-306.

Ward, G. e B. Birner (2001): Discourse and information structure. Evanston: Northwestern University. $<$ http://www.ling.northwestern.edu/ ward/HDA01.pdf > . Acedido em 28-092013.

Recebido: 15 setembro 2014

Aceitado: 16 outubro 2014

Revisto: 13 novembro de 2014

Publicado: 22 junho 2015

Atualizado: 24 junho 2015 